\title{
The dark halo in the elliptical galaxy NGC 3108
}

Gyula I. G. Józsa

RAIUB, Auf dem Hügel 71, D-53121 Bonn, Germany

Thomas A. Oosterloo and Raffaella Morganti

ASTRON, P.O. Box 2, 7990 AA Dwingeloo, The Netherlands

Daniela Vergani

Observatoire de Paris - Section de Meudon, 5, Place Jules Janssen, F-92195 Meudon Cedex, France

\begin{abstract}
We investigate the properties of the dark halo of the dustlane elliptical galaxy NGC 3108. This galaxy contains a large massive gaseous disk, which shows rapid and regular rotation. That makes NGC 3108 a target for measurements usually feasible only for spriral galaxies. Combining spectroscopic measurements of the disk in the opticaland the radio regime, we provide a reliable rotation curve of the galaxy out to $3 R_{\text {eff }}$ and show that the rotation curve does not decline up to a radius of $5.5 R_{\text {eff }}$ corresponding to $28 \mathrm{kpc}$.
\end{abstract}

\section{Observations and data reduction}

In the outer parts the disk of NGC 3108 consists of neutral hydrogen which starts to become ionised towards the centre as already noticed in former observations (Caldwell 1984, Oosterloo et al. 2002). At a distance of $1 R_{\text {eff }} \equiv 5.2 \mathrm{kpc}$ the disk is completely ionised. It extends out to a radius of about $5.5 R_{\text {eff }}$ and shows a symmetric warp starting at about $3 R_{\text {eff }}$.

For our analysis we used spectroscopic observations in the optical and the radio regime. Long-slit-spectroscopy was performed with the La Silla $3.6 \mathrm{~m}$ optical telescope in 1999 including the $\mathrm{H} \alpha$, [NII](6583), and [OIII](5006) emission lines. Radio observations of the HI emission line were carried out by means of synthesis observations with the VLA in 1998.

Using the Karma- and the GIPSY software packages, we extracted two rotation curves. One was found by analysing the line profiles along a fixed straight line aligned with the inner disk. The second rotation curve resulted from the additional analysis of the line profiles along two outer straight lines following the ridge of the warp. The optical rotation curves were derived by fitting full Gaussians to the line profiles, while the HI profiles were fitted with half-Gaussians at the extreme velocity side (following García-Ruiz 2001). The HI- and optical rotation curves were concatenated at a radius of $12^{\prime \prime}$ and the velocities were corrected for an inclination of $75^{\circ}$ as derived by Caldwell (1984). 


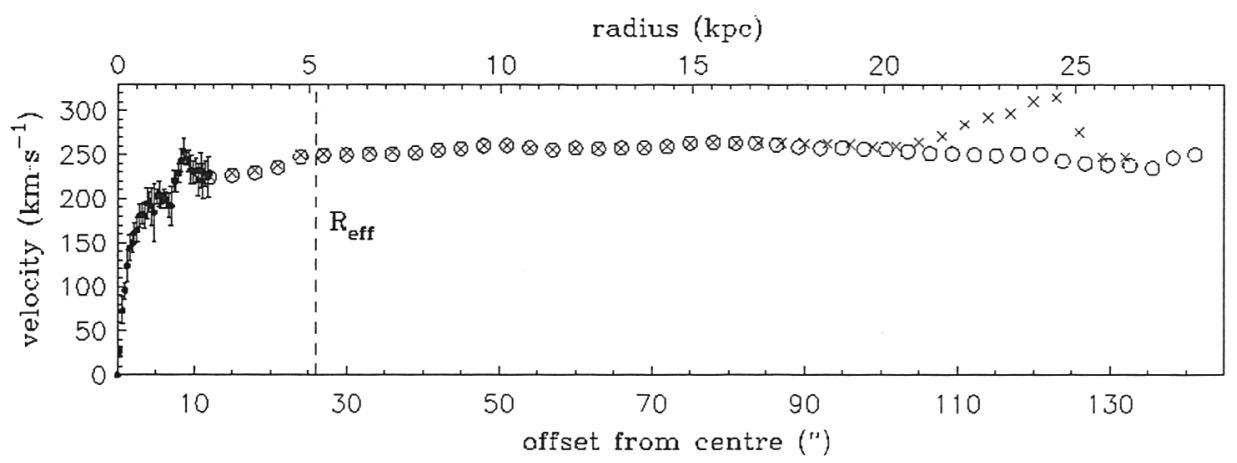

Figure 1. Rotation curves of NGC 3108. Dots denote the optical rotation curve, crosses the $\mathrm{HI}$ rotation curve evaluated along the major axis of the inner disk $\left(\Delta v=3.7 \mathrm{~km} \mathrm{~s}^{-1}\right)$, open circles the HI rotation curve evaluated following the ridge of the warp $\left(\Delta v=5.5 \mathrm{~km} \mathrm{~s}^{-1}\right)$.

\section{Results and discussion}

Fig. 1 shows an overlay of the two derived rotation curves. After a steep central rise they stay flat far beyond the stellar main body, indicating that the galaxy is embedded in an extended massive Dark Matter halo. Within a radius of $3 R_{\text {eff }}$ both rotation curves coincide as the disk shows no kinematic and morphological peculiarities. The rotation curve following the projected warp stays flat out to our last measured point. However, the rotation velocities show a steep increase when tracking the line profiles along a fixed (inner) position angle. As this rise corresponds to a symmetric feature in the kinematics, it is unlikely to be due to the fact that the gas has not settled on stable orbits in the region of the warp. The rise could be produced by a large, unconsidered variation of the inclination $i$ of the disk at smaller radii, or by a real change of the gravitational potential with height above the plane defined by the inner disk (resulting in non-circular motions). Assuming the orbits to be circular, we can derive a lower limit of the dynamical mass corresponding to the datapoint with the highest velocity with respect to the center. At a distance of $4.7 R_{\text {eff }}$ from the center we estimate a dynamical mass of $5.5 \cdot 10^{11} \mathrm{M}_{\odot} \sin ^{-2} i$ and a mass-to-light ratio of $17 \mathrm{M}_{\odot} / \mathrm{L}_{\odot}^{\mathrm{B}} \sin ^{-2} i$.

NGC 3108 is embedded in a massive extended DM halo and our derived rotation curves (assuming orbital circularity) do not show a Keplerian falloff towards the last measured points; rather, they stay flat or even show a rise.

\section{References}

Caldwell, N. 1984, ApJ, 278, 96

García-Ruiz, I. 2001, PhD Thesis, Univ. Groningen

Oosterloo, T. A., Morganti, R., Sadler, E., Vergani, D., Caldwell, N. 2002, AJ, 123,729 